\title{
Dose efficace liée à la consommation d'eau minérale naturelle par l'adulte et le nourrisson*
}

\author{
H. MÉTIVIER**,***, M. ROY**,***
}

(Manuscrit reçu le 23 juin 1997, accepté le I2 septembre 1997)

RÉSUMÉ Les Français consomment régulièrement des eaux minérales en provenance de régions où la concentration de radionucléides naturels est significative. En prenant les derniers coefficients de doses calculés en fonction de l'âge par la Commission Internationale de Protection Radiologique (Publications 56, 67, 69 et 72), il est montré que les eaux naturelles de grande consommation se classent en deux catégories : les eaux non gazeuses qui, pour une consommation exclusive pendant une année, délivreraient une dose efficace engagée voisine de $0,03 \mathrm{mSv}$ et les eaux minérales gazeuses et salines qui délivreraient des doses 10 à 40 fois plus fortes. Pour le nourrisson dont l'alimentation est très souvent préparée à l'aide d'eaux minérales de la première catégorie, la dose efficace engagée jusqu'à l'âge de 70 ans qui résulterait d'une consommation exclusive pendant la première année serait voisine de $0,3 \mathrm{mSv}$.

ABSTRACT Effective doses from natural spring waters ingested by adults and infants. People in France drink commonly mineral spring waters from those regions where concentrations of natural radionuclides are high. Using the recently updated age dependent dose coefficients recommended by the International Commission on Radiological Protection (Publications 56, 67, 69, and 72), it is shown that the largely consumed natural waters can be separated into two categories : non gaseous waters for which a constant exclusive consumption would result into an anmual committed effective dose around than $0.03 \mathrm{mSv}$; and the saline and gaseous ones for which, in same conditions, the dose would be 10 to 40 times higher. For the infant, the diet of whom is often including largely the first category, the annual effective dose committed over 70 years of lifetime would be around than $0.3 \mathrm{mSv}$.

Les radionucléides naturels présents dans l'environnement peuvent se retrouver en proportions variables dans les eaux minérales. Ceux que l'on rencontre le plus souvent sont tous les éléments des familles de l'uranium, du radium et du thorium présents dans les roches traversées par ces eaux. Parmi ces éléments, le radium et les descendants solides du radon, en particulier le plomb 210 et le polonium 210 tiennent avec l'uranium et plus rarement le thorium une place importante (Jeanmaire, 1984). Bien que généralement peu solubles, ils

\footnotetext{
* Article paraissant en publication accélérée.

** Institut de protection et de sûreté nucléaire, BP 6, 92265 Fontenay-aux-Roses cedex, France.

*** Membre du Comité 2 de la commission internationale de protection radiologique.
} 
sont néanmoins présents à l'état de traces et ingérés par les consommateurs de ces eaux en quantités significatives, comme l'ont montré J.C. Harduin et al. (1994) pour l'uranium dans l'eau de Badoit.

À partir des mesures de radioactivité effectuées à l'époque par le Service Central de Protection contre les Rayonnements Ionisants ${ }^{(1)}$, (SCPRI) sur les eaux minérales françaises (Rémy et Pellerin, 1982; Remy et Lemaître, 1990) nous avons calculé les doses engagées que recevraient les consommateurs ne buvant que des eaux minérales. Les résultats montrent qu'elles seraient d'environ $0,3 \mathrm{mSv}$ par an, pour l'adulte et pour le nourrisson. Ces valeurs sont à mettre en perspective avec la valeur de $3 \mathrm{mSv}$ délivrée par l'irradiation naturelle (UNSCEAR, 1993) et celle de $0,1 \mathrm{mSv}$ recommandée par l'Organisation Mondiale de la Santé, (WHO, 1993) pour la radioactivité ajoutée aux eaux de boissons par les diverses activités humaines.

\section{Méthodologie}

\subsection{Quantités de radionucléides dans les eaux minérales françaises}

Les mesures publiées par le SCPRI, concernent 255 eaux de sources dont 26 plus couramment consommées dans notre pays (Remy et Pellerin, 1982; Remy et Lemaitre, 1990). Elles ont porté sur les activités $\alpha$ et $\beta$ totales et sur l'activité en radium 226, sur les masses d'uranium et de thorium naturels ainsi que sur le potassium, dont on peut déduire les activités de certains des descendants solides du radon comme le polonium 210 et le plomb 210 . En effet, les hypothèses de calcul permettent d'évaluer, à partir de l'activité $\beta$ globale, de celles du potassium 40 et du radium 226, et à partir du radium 226 celles de ses descendants, polonium 210 et plomb 210 . Les limites de détection indiquées par les auteurs sont : pour l'uranium de $0,5 \mu \mathrm{g}^{-1}$ ou $12,5 \mathrm{mBq}^{-1}$; pour le radium $226 \mathrm{de} 30 \mathrm{mBq} \mathrm{l}^{-1}$; pour le radium $228 \mathrm{de} 100 \mathrm{mBq}^{-1}$; et pour le thorium de $10 \mu \mathrm{g} \mathrm{l}^{-1}$. Les eaux étudiées contenait rarement une masse mesurable de thorium naturel car il est très peu soluble. Seules les eaux Saint Yorre et Badoit, contenaient des activités mesurables de thorium 228 dont la présence a été attribuée à celle de quantités significatives de son père, le radium 228 . Les valeurs des activités de thorium 230 n'ont pas été retenues ici car, selon les auteurs, elle présentent des erreurs importantes en raison de leur faible niveau.

Parmi les eaux étudiées, on peut en distinguer deux catégories, n'ayant pas les mêmes propriétés et conduisant à des consommations de natures différentes : - Les eaux non gazeuses, peu salines sont largement consommées comme eaux de boisson courantes : par exemple, Evian, Volvic, Vittel et Contrexéville, les trois premières servant aussi très souvent, à préparer les biberons des

(1) Le SCPRI est devenu en 1994 l'OPRI, Office de Protection contre les Rayonnements lonisants. 
nourrissons. Elles ne contiennent que des traces de radionucléides qui restent cependant mesurables.

- Les eaux gazeuses, et salines, sont consommées par les adultes mais jamais par les nouveau nés. C'est le cas des eaux de Badoit, Vichy, Saint Alban, etc. Ces eaux contiennent des quantités plus importantes de radionucléides d'origine naturelle. L'eau Perrier qui est l'exemple d'une eau gazeuse, non saline, en contient beaucoup moins, et pourrait être rattachée à la catégorie précédente.

\section{TABLEAU I}

Activité de plusieurs eaux minérales françaises $\left(\mathrm{mBq} \mathrm{I}^{-1}\right)$. Les valeurs sont déduites des mesures publiées par Remy et Lemaitre, (1990).

Activity of some french natural spring waters $\left(\mathrm{mBq} \mathrm{I}^{-1}\right)$ Values are after measurements published by Remy et Lemaitre, (1990).

\begin{tabular}{|c|c|c|c|c|c|c|c|c|}
\hline $\begin{array}{l}\text { Radio- } \\
\text { nucleide }\end{array}$ & $\begin{array}{l}\text { Evian } \\
\text { Cachat }\end{array}$ & Volvic & $\begin{array}{l}\text { Con-: } \\
\text { trexé- } \\
\text { ville }\end{array}$ & Vittel & $\begin{array}{l}\text { Perrier } \\
\text { Vergèze }\end{array}$ & $\begin{array}{l}\text { St } \\
\text { Yorre } \\
\text { Royale }\end{array}$ & $\begin{array}{l}\text { Badloit } \\
\text { St Gal- } \\
\text { mier }\end{array}$ & Alban \\
\hline${ }^{226} \mathrm{Ra}^{1}$ & 40 & 40 & 27 & 29 & 26 & 250 & 240 & 1800 \\
\hline${ }^{228} \mathrm{Ra}^{2}$ & 4 & 42,7 & 2,9 & 2,6 & 500 & 170 & & \\
\hline${ }^{234} \mathrm{U}$ & 15 & 12 & 19 & 15 & 40 & 130 & 2500 & \\
\hline${ }^{235} \mathrm{U}$ & & & & & & & 44 & \\
\hline${ }^{238} \mathrm{U}$ & 15 & 12 & 19 & 15 & 40 & 26 & 900 & 305 \\
\hline${ }^{210} \mathrm{~Pb}^{3}$ & 28 & 28 & 19 & 20 & 18 & 175 & 168 & 1260 \\
\hline${ }^{210} \mathrm{Po}^{4}$ & 16 & 16 & 10 & 11 & 10 & 100 & 96 & 720 \\
\hline${ }^{228} \mathrm{Th}^{5}$ & & & & & & 28 & 14 & \\
\hline
\end{tabular}

1. Activités supposées égales à la limite de détection.

2. ${ }^{228} \mathrm{Ra}$ (hypothèse pessimiste de $10 \%$ de l'activité ${ }^{226} \mathrm{Ra}$ ).

3. ${ }^{210} \mathrm{~Pb}$ (activité égale à 0,7 activité ${ }^{226} \mathrm{Ra}$ ).

4. ${ }^{210} \mathrm{Po}$ (activité égale à 0,4 activité ${ }^{226} \mathrm{Ra}$ ).

5. Thorium naturel : aucune des sources étudiées ne présente d'activité supérieure à la limite de détection. Seules les eaux de St Yorre et Badoit contiennent du ${ }^{228} \mathrm{Th}$, par la décroissance du ${ }^{228} \mathrm{Ra}$ qui est trés faiblement soluble.

Nous n'avons retenu dans cette étude que quelques eaux largement diffusées et consommées sur le territoire national ainsi qu'à l'étranger, représentatives de leur groupe ou de leur type de consommation. L'activité des différents radionucléides naturels que contiennent les plus courantes d'entre ces eaux sont résumées dans le tableau. Les valeurs proviennent des mesures faites par le SCPRI que nous tenons comme références. Quelques hypothèses de calcul déduites des informations apportées par Remy et Lemaitre, ont permis d'évaluer à partir de l'activité en radium total celle des isotopes 226 et 228 et les descendants solides du radon 222 polonium et plomb 210 , l'activité de 
l'uranium total, à partir de sa masse, et dans quelques cas les différents isotopes de celui-ci. Le thorium n'a pas été retenu dans la plupart des cas, sauf pour le thorium 228 des sources Saint Yorre et Badoit.

\subsection{Coefficients de doses par unités d'incorporation en fonction de l'âge}

La Commission Internationale de Protection Radiologique (CIPR), dans ses publications (ICRP, 1989; ICRP, 1993; ICRP, 1995) compilées dans la publication (ICRP, 1996), recommande pour le public à différents âges des coefficients de dose efficace engagée Par Unité d'Incorporation, (DPUI en Sv Bq-1). L'engagement de la dose est fait pour 50 ans chez l'adulte et jusqu'à l'âge de 70 ans pour le nourrisson. La CIPR recommande que la valeur du nourrisson de trois mois soit appliquée durant toute la première année de la vie. Ces coefficients, pour un même becquerel ingéré de certains radionucléides (radium et thorium), sont 10 à 50 fois plus élevés pour les enfants que pour les adultes et plus particulièrement pour les nourrissons. Ceci est lié à plusieurs facteurs : la masse corporelle plus petite, l'affinité importante de ces éléments pour des organes cibles tels que l'os à métabolisme très actif chez l'enfant, et surtout celle des actinides, un facteur d'absorption digestive environ 10 fois plus élevé pour les nourrissons que pour les adultes. Dès la deuxième année ces coefficients de doses diminuent régulièrement jusqu'à l'âge adulte (Tab. II). Ces valeurs de DPUI ont été reprises dans la Directive Européenne du 13 mai 1996.

\section{TABLEAU II}

Effet de l'âge sur la dose efficace (Sv $\mathrm{Bq}^{-1}$ ) par ingestion, (CIPR 72, 1996). $R=$ DPUI Enfant/DPUI Adulte.

Age dependency of the effective dose ( $\left.\mathrm{Sv} \mathrm{Bq}^{-1}\right)$ from ingestion, (CIPR 72, 1996$)$. $R=$ DPUI child/DPUI Adult.

\begin{tabular}{|c|c|c|c|c|c|c|}
\hline $\begin{array}{l}\text { Radior } \\
\text { nuclaide }\end{array}$ & $\begin{array}{l}\text { Drul } \\
\text { Adulte } \\
\text { Sv Aq }\end{array}$ & 3 mois & $1 \mathrm{an}$ & S ans & 10 & $\begin{array}{c}R \\
15 \text { ans }\end{array}$ \\
\hline${ }^{210} \mathrm{~Pb}$ & $6,9 \times 10^{-7}$ & 12,2 & 5,2 & 3,2 & 2,8 & 2,8 \\
\hline${ }^{210} \mathrm{Po}$ & $1,2 \times 10^{-6}$ & 21,7 & 7,3 & 3,7 & 2,2 & 1,3 \\
\hline${ }^{226} \mathrm{Ra}$ & $2,8 \times 10^{-7}$ & 16,8 & 3,4 & 2,2 & 2,9 & 5,4 \\
\hline${ }^{228} \mathrm{Ra}$ & $6,9 \times 10^{-7}$ & 43,5 & 8,3 & 4,9 & 5,7 & 7,7 \\
\hline${ }^{228} \mathrm{Th}$ & $7,2 \times 10^{-8}$ & 51,4 & 5,1 & 3,1 & 2,1 & 1,3 \\
\hline${ }^{234} \mathrm{U}$ & $4,9 \times 10^{-8}$ & 7,6 & 2,7 & 1,8 & 1,5 & 1,5 \\
\hline${ }^{235} \mathrm{U}$ & $4,7 \times 10^{-8}$ & 7,5 & 2,8 & 1,8 & 1,5 & 1,5 \\
\hline${ }^{238} \mathrm{U}^{\mathrm{U}}$ & $4,5 \times 10^{-8}$ & 7,6 & 2,7 & 1,8 & 1,5 & 1,5 \\
\hline
\end{tabular}




\subsection{Régimes alimentaires adoptés}

Un homme adulte standard absorbe, selon les auteurs, un maximum de 2,5 litres (ICRP 23, 1975; Monod et Flandrois, 1994) à 3 litres (Guénard, 1991) d'eau par jour sous formes diverses. Ces auteurs considèrent que la moitié est constituée d'eau de boisson, celle-ci pourrait donc atteindre en moyenne 1,5 litre par jour soit 547 litres par an, et même davantage chez les gens qui se soumettent à certains régimes.

Chez le nourrisson de 0 à 1 an, le poids de lait et de boisson ingérés par 24 heures est, (selon Ferrier, 1978) de un sixième à un dixième du poids corporel augmenté de $300 \mathrm{~g}$. Les poids de référence étant de $3 \mathrm{~kg}$ à la naissance et de $10 \mathrm{~kg}$ à 1 an (ICPR, 1994), on peut considérer que la consommation moyenne est de 1 litre d'eau par jour, soit 365 litres pendant la première année de la vie.

\section{Résultats}

Pour l'eau d'Evian, la contribution respective de chaque radionucléide dans le calcul de la dose efficace engagée pour une consommation d'eau minérale pendant un an est détaillée pour l'adulte et le nourrisson jusqu'à l'âge de un an dans le tableau III.

\section{TABLEAU III}

Dose efficace engagée par l'ingestion journalière d'eau d'Evian pendant un an.

Committed effective dose from daily ingestion of Evian water during one year.

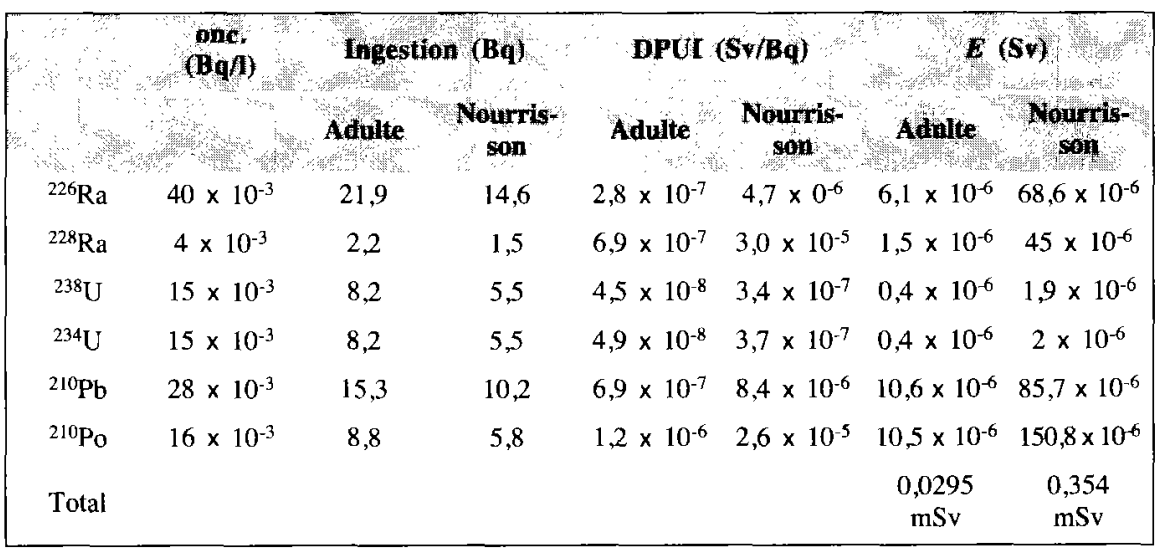

Ces calculs montrent que si cette eau qui contient seulement des traces de radioactivité naturelle, était consommée quotidiennement et exclusivement pen- 
dant toute l'année comme eau de boisson ou pour préparer les biberons, la dose efficace annuelle engagée sur 50 ans qui résulterait de cette ingestion par un adulte serait voisine de $0,03 \mathrm{mSv}$. Pour un nourrisson, la dose engagée jusqu'à l'âge de 70 ans par la consommation durant la première année de la vie serait voisine de $0,35 \mathrm{mSv}$.

\section{TABLEAU IV}

Dose efficace engagée $(\mathrm{mSv})$ par la consommation pendant un an de quatre eaux minérales calculées avec la méthodologie définie pour l'eau d'Evian.

Committed effective doses ( $\mathrm{mSv}$ ) from one year daily ingestion of four spring waters calculated with the Evian defined method.

\begin{tabular}{|ccccc|}
\hline & ntan & Volve & Contrexevine & Vitte \\
Adulte & 0,03 & 0,03 & 0,02 & 0,02 \\
Nourrisson & 0,35 & 0,35 & 0,24 & 0,26 \\
\hline
\end{tabular}

Dans le tableau IV sont comparées les doses efficaces engagées par une consommation annuelle d'eaux de Volvic, Contrexeville et Vittel calculées selon la même méthode que celle utilisée pour l'eau d'Evian. Les valeurs obtenues pour les quatre sources sont très proches.

Pour les eaux gazeuses et salines diverses, il est inutile de calculer la dose à l'enfant car les propriétés organoleptiques de ces eaux les excluent de la consommation par cette partie du public. Il en sera de même pour les nourrissons pour qui la consommation d'eau gazeuse n'est pas vraisemblable.

Les valeurs de doses annuelles calculées pour une consommation quotidienne et exclusive d'eau Perrier serait proche de celles des eaux non gazeuses. Pour Vichy et Badoit, les doses délivrées par ces eaux gazeuses seraient de l'ordre du tiers de mSv. Rappelons que l'Organisation Mondiale de le Santé, recommande pour l'eau de boisson une limite annuelle de $0,1 \mathrm{mSv}$. Des cas extrêmes de certaines eaux riches en radium et uranium naturels peuvent être rencontrées en France (St Alban). Un adulte, qui en ferait son eau de boisson quotidienne et exclusive pendant un an, recevrait une dose engagée voisine de $1 \mathrm{mSv}$. Des valeurs plus élevées ont été mesurées en Chine (Zhang et al., 1994) pour certaines eaux naturelles comme celle de la province de Shandong, cependant il s'agit dans ce cas de sources chaudes qui ne sont probablement pas habituellement consommées. On retrouve, par contre, des valeurs comparables dans les eaux minérales allemandes (Gans et al., 1987) et italiennes (Cigna et Talenti, 1965). 
TABLEAU $\mathrm{V}$

Dose efficace engagée annuelle (mSv) par l'ingestion journalière de quatre eaux minérales gazeuses et salines par un adulte.

Committed effective doses (mSv) from one year daily ingestion of four spring fizzy waters by an adult.

\begin{tabular}{|ccccc|}
\hline Sources & Perrier & Vichy & Badoit & St Alhan \\
Adulte & Vergeze & St Yorre & St Galmier & \\
\hline
\end{tabular}

\section{Incertitudes liées à ces calculs de doses}

Il existe des incertitudes pour les mesures d'activité, comme pour toute mesure. Cependant elles sont faibles car elles sont faites avec des méthodes bien établies et fiables et on sait que les concentrations en ions de ces eaux qui résultent d'un très long échange entre les eaux et les minéraux, sont stables. Un degré d'incertitude demeure cependant lors de l'interprétation des valeurs inférieures à la limite de détection.

Les incertitudes de la dosimétrie sont surtout liées aux simplifications inhérentes à la modélisation, à l'extrapolation de données animales à des valeurs probables chez l'homme et à l'adoption de valeurs moyennes pour les paramètres de transfert. Elles sont souvent plus grandes pour l'enfant que pour l'adulte. Une étude de sensibilité non publiée réalisée pour le compte de la CIPR a évalué l'intervalle de confiance du facteur de transfert gastro intestinal pour un certain nombre de radionucléides. Ces valeurs sont fiables puisqu'elles ont pour la plupart des éléments étudiés, été obtenues chez l'homme, et pour l'uranium, directement à partir de l'eau de Badoit (Harduin et al., 1994). Pour le nourrisson, cependant, l'incertitude sera plus grande puisque ces coefficients de transfert ont été majorés par extrapolation selon une règle établie par un groupe d'expert de l'AEN (1988), et repris par la CIPR, en s'appuyant sur des mesures chez le primate non humain. Ces mesures ont largement contribué à l'établissement de cette règle. On sait aussi que pour nombre de ces éléments le transfert gastro intestinal est augmenté lorsque le consommateur est à jeun, comme c'est le cas des personnes qui consomment de l'eau minérale durant la nuit ou au réveil, lors d'une cure, par exemple.

Restent enfin, deux incertitudes liées au modèle digestif lui même. La première est la dose délivrée au niveau de la bouche absente du modèle actuel. En effet, il a été montré qu'une consommation régulière de plutonium soluble sous forme de carbonate dans l'eau pouvait chez le rat conduire à une fixation importante du radioélément au niveau du collet, ou de la plaque dentaire (Renaud-Salis et al. 1990). Cette observation a été récemment confirmée pour 
les métaux lourds, avec le plomb, chez l'homme (Gil et al., 1996). Cette fixation directe observée pour les métaux lourds est plus que probable dans notre étude pour l'adulte. La seconde incertitude est liée au calcul de la dose au niveau intestinal où la dose aux tissus dépend du régime alimentaire et qui, selon J. Stubbs (1997), pourrait être surestimée d'un facteur 2.

Une estimation globale des incertitudes liées à ces calculs reste difficile, cependant les différents éléments d'appréciation dont on dispose, permettent de donner pour les doses obtenues, un facteur de fiabilité de 2 à 3 aussi bien pour l'enfant que pour l'adulte.

\section{Conclusion}

Certaines eaux minérales gazeuses contiennent une radioactivité naturelle entraînant une irradiation interne lorsqu'elles sont bues régulièrement en grande quantité. Elles conduisent si elles sont consommées exclusivement à des doses voisines de $0,3 \mathrm{mSv}$. Ces eaux sont aussi riches en sels minéraux et en gaz dissous et sont essentiellement consommées uniquement par des adultes, mais jamais par des nouveau-nés et rarement chez les enfants. Au contraire, beaucoup d'eaux minérales faiblement minéralisées et non gazeuses, comme Evian, Vittel, Contrexéville, Volvic, peuvent être absorbées en ration liquide quotidienne par le très jeune enfant, au moins pendant la première année de sa vie. En dépit de la valeur élevée chez celui-ci des doses efficaces engagées par unité d'incorporation, la dose engagée jusqu'à l'âge de 70 ans par une consommation quotidienne et exclusive serait inférieure à $0,35 \mathrm{mSv}$. Une comparaison avec d'autres eaux minérales européennes montrent que ces doses, composantes de l'irradiation totale liée à la radioactivité naturelle sont sensiblement égales pour chaque type d'eau minérale et chaque habitude de consommation. Ces valeurs sont à comparer aux autres composantes de l'irradiation naturelle qui atteint en France une valeur proche de $3 \mathrm{mSv}$ par an.

\section{RÉFÉRENCES}

Cigna A.A. and Talenti M. (1965) La radioattivita naturale di alcune acqua minerale Italiane, Minerva Nucleare, 9, 248-250.

Directive 96/29 Euratom du Conseil du 13 mai 1996, fixant les normes de base relatives à la protection sanitaire de la population et des travailleurs contre les dangers résultant des rayonnements ionisants. J.O.C.E. n 159 du 29 juin 1996.

Ferrier P. (1978) Précis de Pédiatrie (Payot, Paris, $2^{\mathrm{c}}$ Edition).

Gans I. (1985) Natural radionuclides in mineral waters. Sci. total Environm. 45, 93-99. 
Gans I., Fusban H.U., Wollenhaupt H., Kiefer J, Glöbel B., Berlich J., Postendörfer J. (1987) Radium 226 und andere natürliche Radionuklide im Trinkwasser und in Getränken in der Bundesrepublik Deutschland. Institut für Wasser-, Boden- und Lufthygiene des Bundesgesundheitsamtes. Berlin (WaBoLu 4/1987).

Gil F., Facio A., Villanueva E., Pérez M.L., Tojo R., Gil A. (1996), The association of tooth lead content with dental health factors, Sci. total Environnment, 192, 183-191.

Guenard H. (1991) Précis Physiologie Humaine (Pradel, Paris).

Harduin J.C., Royer P. and Piechowski J. (1994) Uptake and Urinary Excretion of Uranium after Oral Administration in Man. Rad. Prot. Dosim. 53, 1-4, 245-248.

ICRP (1975), Report of a Task Group on Reference Man. (Publication 23) Oxford, Pergamon Press.

ICRP (1989), Age Dependent Doses to Members of the Public from Intake of Radionuclides; Part 1 (Publication 56) Oxford, Pergamon Press.

ICRP (1993), Age Dependent Doses to Members of the Public from Intake of Radionuclides : Part 2 Ingestion dose coefficients, (Publication 67), Oxford Elsevier Science Ltd.

ICRP (1994), Human respiratory tract model for radiological protection, (Publication 66), Oxford Elsevier Science Ltd.

ICRP (1995), Age Dependent Doses to Members of the Public from Intake of Radionuclides : Part 4 Ingestion dose coefficients, (Publication 69), Oxford Elsevier Science Ltd.

ICRP (1996), Age Dependent Doses to Members of the Public from Intake of Radionuclides : Part 5 Compilation of ingestion and Inhalation dose coefficients, (Publication 72), Oxford Elsevier Science Ltd.

Jeanmaire L. (1994), Radioactivité naturelle des aliments, In : Proceedings of the International Radiological Protection Association Congress IRPA 6. Sydney.180-184.

Monod H. et Flandrois R. (1994), Physiologie du Sport (Masson, Paris).

NEA/OECD (1988) Committee on Radiation Protection and Public Health. report of an Expert Group on Gut Transfer factors, NEA/OECD Report, Paris.

Remy M. L, Pellerin P. (1982) Quelques données récentes sur la radioactivité naturelle des sources hydrominérales françaises, Presse Thermale et Climatique, 119, 3, 150-155.

Remy M.L., Lemaitre N. (1990) Eaux Minérales et Radioactivité; Hydrogéologie, 4, 267-278.

Renaud-Salis V., Lataillade G., Métivier H., (1990) Effect of mass, oxidation state and duration of chronic ingestion of plutonium absorption in fead rats. Int J. Radiat Biol, 58, 4, 691-704.

Stubbs J. (1997) Communication personnelle.

UNSCEAR (1993) Report to the general Assembly with Annexes. United Nations Scientific Committee on the Effects on Atomic Radiation Sources and Biological Effects (United Nations, New York)

WHO (1993), Guidelines for drinking water quality. World Health Organization, Second edition. Vol1. WHO, Genève.

Zhang Meiying, Wang Wentuan and Geng Ming. (1994) Investigation of natural radioactivity level of the waters in Shandong province. Radiation Protection (en Chinois), 14, 284-289. 